I Sección de Historia: Centenario de la Revolución Rusa 1917-2017

\title{
El desarrollo científico y tecnológico en la Unión Soviética (1917-1991): Una sinopsis
}

\author{
Ronald Eduardo Díaz Bolaños \\ Universidad Estatal a Distancia, Costa Rica \\ ronald.diaz@ucr.ac.cr \\ https://orcid.org/0000-0003-2860-6744
}

Recibido: 4 de marzo de 2018

Aceptado: 9 de abril de 2018

\section{RESUMEN}

A lo largo de su existencia, la Unión de Repúblicas Socialistas Soviéticas (URSS) experimentó una serie de transformaciones políticas, económicas y sociales que convirtieron al antiguo imperio ruso zarista, de base económica agrícola, en una superpotencia caracterizada por su progresivo desarrollo industrial. En este proceso, que abarcó desde el triunfo de la Revolución Rusa (1917) hasta la disolución de la Unión Soviética (1991), el desarrollo científico y tecnológico fue fundamental debido al impulso que el Estado soviético brindó a la ciencia y la tecnología en el marco de la construcción de un modelo económico socialista, proceso que experimentó sus contradicciones bajo el estalinismo y que recibió un estímulo significativo con el desarrollo de la carrera armamentista y la exploración espacial durante la Guerra Fría. Los cambios vividos por la Unión Soviética en la década de 1980 también se reflejaron en su desarrollo tecnocientífico.

Palabras claves: Ciencia; Tecnología; Historia; Unión Soviética; Guerra Fría

Scientific and Technological Development in the Soviet Union (1917-1991): A Synopsis

\section{ABSTRACT}

Throughout its existence, the Union of Soviet Socialist Republics (USSR) experienced a series of political, economic and social transformations that

La Revista Estudios es editada por la Universidad de Costa Rica y se distribuye bajo una Licencia Creative Commons Atribución-NoComercial-Compartirlgual 3.0 Costa Rica. Para más información envíe un mensaje a 
Revista Estudios, (36), 2018.

Junio 2018-Noviembre 2018

ISSN 1659-3316

Díaz Bolaños Ronald Eduardo

converted the former Tsarist Russian Empire of agricultural economic base, into a superpower characterized by its progressive industrial development. In this process, which ranged from the triumph of the Russian Revolution (1917) to the dissolution of the Soviet Union (1991), scientific and technological development was fundamental due to the support that the Soviet State provided to science and technology in the construction of a socialist economic model, a process that experienced its contradictions under Stalinism and that received a significant stimulus with the development of the arms race and space exploration during the Cold War. The changes experienced by the Soviet Union in the 1980s were also reflected in its techno-scientific development.

Key words: Science; Technology; History; Soviet Union; Cold War

\section{INTRODUCCIÓN}

Las hazañas realizadas en el Cosmos indicaban con toda diafanidad la madurez de la ciencia y la cultura soviéticas, el elevado poderío económico del país y su lugar en la civilización universal.

El camino de las estrellas tenía su punto de partida en las aulas escolares y estudiantiles, pasaba por los institutos y centros científicos, bibliotecas y museos y se preparaba en los laboratorios, fábricas y minas.

Lelchuk, Poliakov y Protopopov, (1977, p. 390).

El párrafo anterior condensa el nivel que había alcanzado el desarrollo científico y tecnológico en la Unión Soviética en las postrimerías de la década de 1960, como parte de un esfuerzo del primer Estado socialista del planeta en aplicar una política tecnocientífica que desde las instituciones educativas y científicas, colaboró activamente en la transformación del país hasta convertirse en una superpotencia económica que lideró el bloque oriental, surgido al término de la Segunda Guerra Mundial y fue capaz de incursionar en la exploración del espacio exterior, en el lapso del medio siglo transcurrido desde el triunfo de la Revolución Rusa.

\section{(C) $(00$}

La Revista Estudios es editada por la Universidad de Costa Rica y se distribuye bajo una Licencia Creative Commons Atribución-NoComercial-CompartirIgual 3.0 Costa Rica. Para más información envíe un mensaje a 
Precisamente, en el mes de octubre de 1917 (noviembre en el calendario gregoriano porque el juliano estaba vigente en Rusia) se produce la Revolución Rusa que llevó al poder a los revolucionarios bolcheviques liderados por Vladimir Ilich Ulianov (1870-1924), más conocido como Lenin, quien sentó las bases del nuevo Estado soviético que relegó a la historia a la autocracia zarista. Este proceso tuvo profundas repercusiones políticas, sociales y económicas en el otrora Imperio Ruso que a partir de 1922 adopta el nombre oficial de Unión de Repúblicas Socialistas Soviéticas (URSS) y lo mantuvo hasta su disolución en 1991.

El presente trabajo constituye una revisión bibliográfica de varios textos publicados originalmente en la Unión Soviética, especialmente entre los decenios de 1960 y 1980, que se refieren al desarrollo científico y tecnológico de la otrora superpotencia socialista y fueron traducidos al español por algunas editoriales soviéticas. También se consultaron obras relacionadas con el desarrollo histórico de la URSS desde su fundación hasta su disolución, con el fin de extraer información relacionada con el objetivo de esta investigación que consiste en sintetizar los principales aportes de la Unión Soviética en el campo científico y tecnológico a lo largo de su existencia como estado.

El enfoque teórico en que se fundamenta este artículo es el de la Historia Social de la Ciencia que concibe al conocimiento tecnocientífico como un producto del contexto político, económico, social y cultural en el que se desarrolla (Saldaña, 1996). Dicho sea de paso, este enfoque comenzó a plantearse en la Unión Soviética con la presentación del trabajo pionero del físico e historiador de la ciencia soviético Boris Hessen (1893-1936) sobre la física newtoniana en el Segundo Congreso Internacional de Historia de la Ciencia en Londres (1931) (Hessen, 1989; Saldaña, 1989 y Kojevnikov, 2008).

\section{(c) (i) (2)}

La Revista Estudios es editada por la Universidad de Costa Rica y se distribuye bajo una Licencia Creative Commons Atribución-NoComercial-CompartirIgual 3.0 Costa Rica. Para más información envíe un mensaje a 
Un avance de este artículo se presentó como ponencia en el marco del Seminario "La Revolución Rusa y su impacto en el mundo. 1917-2017" organizado por la Cátedra de Historia de la Cultura de la Escuela de Estudios Generales y la Sección de Historia Universal de la Escuela de Historia de la Universidad de Costa Rica, el 3 de octubre de 2017. El autor agradece el apoyo del Programa de Estudios Sociales de la Ciencia, la Técnica y el Medio Ambiente (PESCTMA, VI805-A4-906) del Centro de Investigaciones Geofísicas (CIGEFI) en el desarrollo de la presente investigación, así como al personal de las bibliotecas Carlos Monge Alfaro y Luis Demetrio Tinoco Castro del Sistema de Bibliotecas, Documentación e Información (SIBDI) de la Universidad de Costa Rica por facilitar varias de las fuentes empleadas durante la recopilación bibliográfica. Se agradece también la colaboración brindada por Edgardo Gallo Guzmán y Luis Ricardo Romero Valverde en la búsqueda de publicaciones en línea durante el proceso de investigación.

\section{Aspectos político-institucionales de la ciencia y la tecnología en la Unión Soviética}

La instauración del socialismo en Rusia significó también un cambio en la orientación de la producción científica que se venía desarrollando en el país desde hacía algunos siglos bajo el gobierno de los zares. El mismo Lenin era consciente de este cambio al afirmar: "Antes todo el espíritu humano, todo el genio del hombre creaba sólo para proporcionar a unos pocos todos los frutos de la técnica y de la cultura, privando así a los demás de lo esencial: la educación y el progreso. Ahora, todas las conquistas de la cultura van a convertirse en patrimonio del pueblo entero" (Keldych, 1970, pp. 8-9). Bajo dicha premisa, el naciente Estado soviético buscó desarrollar una política científica y tecnológica enmarcada dentro del sistema socialista que se estaba construyendo y tendría como fin extender los logros tecnocientíficos al alcance de toda la población soviética. 
A nivel institucional, la Academia de la Ciencias de la Unión de Repúblicas Socialistas Soviéticas (ANSSSR) se constituyó en la principal entidad científica y estuvo supeditada al Estado soviético que subvencionó sus labores (Kara-Murza, 1989). La ANSSSR surgió a partir del proceso de reestructuración que tuvo lugar en la Academia de Ciencias de Rusia en 1925, fundada originalmente con el nombre de Academia Imperial de Ciencias de San Petersburgo por el zar Pedro I el Grande (1672-1725) en 1724, tres décadas antes que se fundara la Universidad de Moscú (1755) y en un contexto en que las condiciones políticas, sociales y económicas prevalecientes en el Estado zarista permitieron el surgimiento de una pequeña comunidad científica rusa (Seton-Watson, 1955 y Balashova, 2016). Esta institución, a la que en 1747 se le agregó la Biblioteca Imperial de San Petersburgo, patrocinó el desarrollo de la actividad científica de la Rusia zarista que organizó exploraciones geográficas en el vasto territorio del Imperio Ruso (Leonov, 2014). Entre los científicos más destacados que colaboraron con la Academia de Ciencias en sus primeras décadas se encuentran el enciclopedista ilustrado Mijail Lomonósov (1711-1765), fundador de la Universidad de Moscú (actualmente lleva su nombre) y el matemático suizo Leonhard Euler (1707-1783) (Mora, 1985 y Pashuto et al., 1985).

A inicios del siglo $X X$, la enorme extensión del imperio ruso, escasamente integrado y con regiones todavía por explorar, constituía un desafío para el nuevo gobierno bolchevique, aunado también al rezago económico con respecto a las potencias industriales europeas: "solamente una pequeña parte de éste [territorio] era apropiada para la agricultura, y bastantes de estos sectores agrícolas tenían un suelo o un clima pobres [...] antes de la Primera Guerra Mundial solo se había desarrollado adecuadamente su petróleo y algunos otros minerales" (Black, 1969, p. 203).

\section{(c) (†) (-)}

La Revista Estudios es editada por la Universidad de Costa Rica y se distribuye bajo una Licencia Creative Commons Atribución-NoComercial-CompartirIgual 3.0 Costa Rica. Para más información envíe un mensaje a 
En la década de 1920, el gobierno revolucionario incluyó la participación de científicos y técnicos de diversos sectores políticos, en cuenta aquellos que no militaron en las filas bolcheviques, para contribuir en la transformación de la sociedad postrevolucionaria, entre ellos el químico Vladimir Ipatieff (1867-1952), simpatizante de la monarquía y artífice de la nacionalización de la industria química y la que se orientaba hacia la investigación en el plano militar; al geoquímico liberal Vladimir Vernadsky (1863-1945), fundador de la Academia de Ciencias de Ucrania y promotor de las campañas de exploración geológicas soviéticas en el extremo nororiental del país. Destacó también el ingeniero eléctrico y militante comunista Gleb Krzhizhanovsky (1872-1959), artífice del proyecto de la Comisión Estatal para la Electrificación de Rusia (GOELRO) cuyo fin era la expansión de la energía eléctrica en el país y que fue adoptado en forma entusiasta por el gobierno de Lenin (Kojevnikov, 2008).

El mismo Lenin había propiciado encuentros con los científicos formados en la Rusia zarista, incluso quienes se habían opuesto a la misma Revolución de 1917 para integrarlos en su proyecto político, que varios de ellos terminaron finalmente de asumir, porque para la transformación de las condiciones económicas y sociales del país bajo un sistema socialista, era necesario el impulso de la ciencia y la tecnología, por eso el primer líder soviético propuso a los miembros de la misma Academia de Ciencias que reorientaran su producción científica al servicio del pueblo soviético (Mora, 1985).

En los primeros años del proceso revolucionario la Academia de Ciencias mantuvo su sede en San Petersburgo (ciudad que se denominó Leningrado entre 1924 y 1991) hasta que en 1934 se trasladó definitivamente a Moscú. El gobierno nacionalizó las fundaciones privadas dedicadas a la investigación científica y fundó una gran cantidad de centros de investigación orientados a dar respuesta a los retos que significaba la modernización del país bajo un modelo socialista; se 
Revista Estudios, (36), 2018.

Junio 2018-Noviembre 2018

ISSN 1659-3316

Díaz Bolaños Ronald Eduardo

trató de reducir la barrera entre la ciencia pura y la ciencia aplicada que se había desarrollado entre las instituciones científicas de los países de Europa Occidental (Kojevnikov, 2008).

Hacia 1940 la Academia de Ciencias de la URSS contaba con 4700 colaboradores científicos y además de su sede moscovita, regentaba 76 institutos, 42 estaciones científicas (biológicas, sismológicas, etc.), 11 laboratorios y 6 observatorios con sus respectivas secciones; además de las academias regionales en Kazajstán, Transcaucasia y los Urales, a las que se sumaron las de Turkmenistán y Uzbekistán. Este conjunto de instituciones le brindó un significativo impulso al desarrollo científico en las zonas rurales y en los territorios de habla no rusa por lo que dieron un especial énfasis a la investigación aplicada y sus aportes al desarrollo del modelo soviético (Lelchuk, Poliakov y Protopopov, 1977 y Pashuto et al., 1985). De acuerdo con Lelchuk, Poliakov y Protopopov (1977, p. 239):

Todas esas instituciones contribuían sustancialmente al desarrollo del pensamiento científico y al empleo de importantes descubrimientos en la industria y la agricultura. Con su ayuda se investigaban los recursos naturales del país, se elaboraban nuevos métodos para su aprovechamiento y se preparaban investigadores.

En la segunda mitad del siglo XX surgen nuevos centros científicos en Novosibirsk (1957), que se constituyó en la filial de la Academia de Ciencias en Siberia; además de los de Tomsk y Vladivostok en Rusia, Donestk y Leópolis en Ucrania (Mora, 1985 y Pashuto et al., 1985). De esta forma, la misma Academia consolidó una red de academias nacionales y regionales y otras de carácter especializado diseminadas a través del territorio soviético y desde donde se ponía en marcha la política estatal en el campo científico y tecnológico. De acuerdo con el matemático Mstislav Keldych (1911-1978), quien fue presidente de la Academia de Ciencias de la Unión Soviética (1966-1975) y uno de los artífices del programa espacial

La Revista Estudios es editada por la Universidad de Costa Rica y se distribuye bajo una Licencia Creative Commons Atribución-NoComercial-CompartirIgual 3.0 Costa Rica. Para más información envíe un mensaje a revistaestudios.eeg@ucr.ac.cr. 
soviético: "Una de las características principales de la ciencia soviética reside en la estrecha relación que establece entre la investigación fundamental y la búsqueda de soluciones prácticas para los problemas económicos" (Keldych, 1970, p. 11).

Al finalizar la década de 1950, la Academia de Ciencias llegó a contar con ocho departamentos, cada uno de los cuales tenía a su cargo diversos institutos de investigación científica y regidos por un Secretariado Científico que informaba a los dirigentes políticos soviéticos sobre los trabajos de la entidad, mientras que la Dirección Superior o Presidium proponía el tipo de investigaciones que debía realizar la Academia. Este último organismo era integrado por sus miembros científicos de más alto rango y entre la comunidad científica soviética -constituida por centenares de miles de especialistas- solamente un puñado contaba con el prestigioso título de académicos, que eran seleccionados entre el personal científico de todas las repúblicas, aunque la mayoría procedía de Moscú o Leningrado (R. de E., 1959).

\section{La ciencia y la tecnológica en la transformación de la sociedad soviética}

A partir de la implantación del socialismo en la naciente Unión Soviética, se planteó como meta la industrialización de la economía para transformar al país en una potencia industrializada, especialmente en el campo de la industria pesada. "Por eso Lenin recalcó la necesidad de construir fábricas, talleres y centrales eléctricas. Se trataba de una tarea ardua y compleja, puesto que Rusia era un país relativamente atrasado" (Lelchuk, Poliakov y Protopopov, 1977, p. 109). Desde sus inicios, las autoridades soviéticas comprendieron las aplicaciones prácticas de la ciencia y la tecnología orientadas hacia la transformación social y económica de un país: "el progreso tecnocientífico [...] no se efectúa aisladamente de la vida social, sino en estrecha conexión con el progreso social” (Tapeznikov, 1974, 9. 63).

\section{(c) (i) (2)}

La Revista Estudios es editada por la Universidad de Costa Rica y se distribuye bajo una Licencia Creative Commons Atribución-NoComercial-CompartirIgual 3.0 Costa Rica. Para más información envíe un mensaje a 
Fue en la segunda mitad de la década de 1920 que comenzó el proceso de transición de la economía soviética hacia una de carácter industrial, fruto de los cambios introducidos por la Nueva Política Económica (NEP) de Lenin y mediante la aplicación del Primer Plan Quinquenal (1928-1932) delineado por su sucesor en el poder lósif Stalin (1878-1953), no sin antes realizar una transformación de las estructuras agrarias heredadas del período zarista.

Paralelamente, se elaboró una política educativa delineada por el mismo Lenin que tenía como meta la eliminación del analfabetismo y el cultivo de la ciencia, en un país donde el $80 \%$ de la población en el rango de edad entre los 9 y 50 años era analfabeta en los albores del siglo XX, porcentaje que aumentaba en los territorios centroasiáticos y siberianos (por ejemplo, tan solo el $0,5 \%$ de los tayikos y el $0,6 \%$ de los kirguises eran alfabetizados). Esta situación llevó a que el mismo Lenin promulgara el Decreto sobre la organización de la Comisión del Estado para la Educación del 22 de noviembre de 1917 y otras leyes posteriores que forjaron el sistema educativo del nuevo Estado, proceso en el que colaboró también Nadezhda Krupskaya (1869-1939), esposa de Lenin, con la publicación de su obra Educación y democracia (1917), por lo que dio inicio al proceso de alfabetización de la población, la expansión de la enseñanza en todos sus niveles y la popularización del conocimiento científico y tecnológico, esfuerzo que fue dificultado por la Guerra Civil Rusa que tuvo lugar entre 1917 y 1922 (Stoletov, 1970).

La aplicación de las ideas de alfabetización y la expansión del sistema educativo soviético se desarrolló en forma paralela con el Primer Plan Quinquenal, que aumentó significativamente la población alfabetizada, inclusive en el Asia Central, de tal forma que entre 1927 y 1932, la proporción de adultos alfabetizados pasó del $4 \%$ al $52 \%$ en Tayikistán y del $12 \%$ al $72 \%$ en Uzbekistán, fruto del esfuerzo 
emprendido por maestros e intelectuales en todo el país (Lelchuk, Poliakov y Protopopov, 1977, p. 174).

La expansión de un sistema escolar gratuito y obligatorio a través del enorme territorio soviético favoreció la enseñanza de la ciencia en las aulas, junto a otros campos del saber como las artes y las letras, aspecto que se vio beneficiado por la extensión de la gratuidad de la enseñanza secundaria -también obligatoria - y la universitaria. Además, como un apoyo al proceso de industrialización, se fomenta la enseñanza técnica, la asignación de becas y franquicias por parte del Estado para quienes debían cursar sus estudios mientras realizaban actividades laborales, por lo que la población con estudios medios y superiores pasó de 15,9 millones en 1939 a 95 millones en 1970 (Efimov, 1982, p. 56).

En esta primera etapa de la industrialización soviética se dio un significativo avance tecnológico con la apertura de la primera central hidroeléctrica soviética en Vóljov (Rusia) en 1926, el envío de expediciones geológicas al macizo de Jibini (Península de Kola), a los Montes Urales y al Asia Central; además de la construcción del complejo metalúrgico de Magnitogorsk (Rusia), el funcionamiento del Turksib, ferrocarril que comunicaba Siberia con los territorios centroasiáticos de Kazajstán y Kirguistán a partir de 1930 y la inauguración de la Central Hidroeléctrica del Dniéper o Dnieprogrés (Ucrania) en 1932. En este último año, por primera vez, fue posible que el rompehielos soviético Sibiriakov viajó entre los puertos de Arcángel y Vladivostok a través de las aguas del Ártico.

En 1935, comenzó a funcionar el Metro de Moscú y dos años más tarde tuvo lugar el primer vuelo entre Moscú y los Estados Unidos a través del Polo Norte, protagonizado por los aviadores Valeri Chkálov (1904-1938) y Mijail Gromov (1899-1985), en la misma época que entraba en operaciones la primera estación soviética en el Polo Norte donde los científicos Evgueni Fiódorov (1910-1981),

\section{(c) (i) (9) (2)}

La Revista Estudios es editada por la Universidad de Costa Rica y se distribuye bajo una Licencia Creative Commons Atribución-NoComercial-CompartirIgual 3.0 Costa Rica. Para más información envíe un mensaje a 
Ernst Krénkel (1903-1971), Iván Papanin (1894-1986) y Piotr Shirshov (19051953) permanecieron durante nueve meses en labores de investigación (Lelchuk, Poliakov y Protopopov, 1977 y Pashuto et al., 1985).

Esta transformación de una sociedad agrícola a una industrial -al menos en el occidente de la Unión Soviética - provocó la admiración del escritor británicoirlandés George Bernard Shaw (1856-1950), quien visitó el país en 1931: "La Rusia del zarismo abominable es ahora un país comunista enérgico, sobrio, puro, intelectual al estilo moderno, independiente, próspero y desinteresado (Lelchuk, Poliakov y Protopopov, 1977, p 159). Todos estos profundos cambios tenían lugar mientras los países capitalistas experimentaban una debacle económica generada por la crisis originada por la quiebra de la Bolsa de Valores de Nueva York en 1929, aunque tampoco estuvieron exentos de contradicciones, por ejemplo el Holodomor, la hambruna que experimentó un sector significativo de la población ucraniana en el marco del proceso de colectivización agrícola a inicios del decenio de 1930, cuyos alcances e implicaciones políticas han sido objeto de debate por parte de la comunidad de académicos (Service, 2010 y Wozniak, 2013).

El Segundo Plan Quinquenal (1933-1937) asignó la cuantiosa suma de 110000 millones de rublos para el fomento de la enseñanza secundaria y universitaria y se financiaron la fundación de nuevas bibliotecas, institutos de investigación y museos. Los avances de la enseñanza permitió la inserción de jóvenes campesinos y obreros a los crecientes cuadros científicos soviéticos como el botánico Vasily F. Kuprevich (1897-1969), quien fue Presidente de la Academia de Ciencias de Bielorrusia y el diseñador de cohetes ucraniano Serguei Koroliov (1907-1966) (Lelchuk, Poliakov y Protopopov, 1977). No obstante, también su aplicación implicó un mayor control de la investigación científica y tecnológica por parte del Estado al sujetarlas a los requerimientos de la planificación central impulsada por los planes quinquenales (Rothbard, 2015).

\section{(c) (i) (2)}

La Revista Estudios es editada por la Universidad de Costa Rica y se distribuye bajo una Licencia Creative Commons Atribución-NoComercial-CompartirIgual 3.0 Costa Rica. Para más información envíe un mensaje a 
El Estado soviético fomentó el reconocimiento de la labor de sus científicos y tecnólogos con la introducción de galardones como el Premio Lenin en 1925 y su posterior sustitución por el Premio Stalin en 1939, al que se la asignó una dotación de 100 mil rublos para cada ganador, entre ellos el destacado diseñador de aviones Serguei lliushin (1894-1977), quien fue premiado en siete ocasiones por su labor en el campo de la aeronáutica. A partir de 1956, con la desestalinización impulsada por el líder soviético Nikita Jruschov (1894-1971), pasa a llamarse nuevamente Premio Lenin, con una dotación de 10 mil rublos y en 1967 se introdujo el Premio del Estado (sustituido en 1992 por el Premio del Estado de la Federación Rusa), cuyo monto asignado a los laureados fue de 5 mil rublos en reconocimiento por sus logros, aunque en una escala menor que los galardonados con el Premio Lenin (Lelchuk, Poliakov y Protopopov, 1977 y Timofeichev, 2017).

La ciencia soviética fue reconocida a nivel internacional con la concesión de los Premio Nobel a connotados científicos soviéticos como lo muestra la tabla 1:

Tabla 1

Científicos soviéticos galardonados con un Premio Nobel

\begin{tabular}{|c|l|l|}
\hline Año & \multicolumn{1}{|c|}{ Nombre } & \multicolumn{1}{|c|}{ Galardón } \\
\hline 1956 & Nikolai Semionov (1896-1986) & Química \\
\hline 1958 & $\begin{array}{l}\text { Pavel Cherenkov (1904-1990) } \\
\text { Iliá Frank (1908-1990) } \\
\text { Igor Tamm (1895-1971) }\end{array}$ & Fisica \\
\hline 1962 & $\begin{array}{l}\text { Lev Davidovich Landau (1908- } \\
\text { 1968) }\end{array}$ & Física \\
\hline 1964 & $\begin{array}{l}\text { Nikolai Basov (1922-2001) } \\
\text { Alexander Projorov (1916- } \\
\text { 2002) }\end{array}$ & Física \\
\hline 1978 & Piotr Kapitsa (1894-1984) & Física \\
\hline
\end{tabular}

Fuente: Lelchuk, Poliakov y Protopopov, 1977; Relación de ganadores de los Premios Nobel (1901-1998) (1999).

La Revista Estudios es editada por la Universidad de Costa Rica y se distribuye bajo una Licencia Creative Commons Atribución-NoComercial-CompartirIgual 3.0 Costa Rica. Para más información envíe un mensaje a revistaestudios.eeg@ucr.ac.cr. 
Los científicos soviéticos comenzaron a participar en congresos internacionales donde dieron a conocer sus aportes e inclusive se organizaron algunos en la misma URSS, entre ellos el XV Congreso Internacional de Fisiología (1935), celebrado en Leningrado y Moscú, bajo la presidencia del célebre fisiólogo Iván Pavlov (1849-1936) y el XVII Congreso Internacional de Geología reunido en Moscú (1937), presidido por el geólogo Iván Gubkin (1871-1939) (Lelchuk, Poliakov y Protopopov, 1977).

El desarrollo tecnológico alcanzado por la Unión Soviética permitió el fomento de la industria militar que fue fundamental para la defensa del territorio soviético durante la Gran Guerra Patria (1941-1945), nombre que recibió la Segunda Guerra Mundial en el contexto soviético y se mantiene en la Rusia contemporánea. En 1942 se produjeron "más de 25.000 aviones, más de 24.000 tanques y unos 57.000 cañones" (Lelchuk, Poliakov y Protopopov, 1977, p. 267) por lo que el Ejército Rojo logró equiparse debidamente para enfrentar los avances del ejército alemán en el occidente del país. Incluso se dio el traslado de varias industrias soviéticas hacia zonas más alejadas del conflicto como la región del Volga, los Urales, Kazajstán y otras repúblicas del Asia Central en los que fueron empleados mujeres, adolescentes y pensionados en sustitución de los obreros movilizados.

Una vez finalizada la guerra, las autoridades soviéticas debieron hacer frente a la rehabilitación de la industria y la infraestructura de los territorios ocupados por el Tercer Reich (entre ellos las repúblicas bálticas de Estonia, Letonia y Lituania, incorporadas a la Unión Soviética en 1940) y muchas fábricas dedicadas a la actividad bélica se dedicaron a la producción de bienes de consumo de carácter civil, por lo que el Cuarto Plan Quinquenal (1946-1950) se orientó a la reactivación económica del país que incluyó la construcción de nuevas centrales hidroeléctricas en Armenia, Georgia y Uzbekistán, impulsar la explotación

\section{(c) (i) (9) (2)}

La Revista Estudios es editada por la Universidad de Costa Rica y se distribuye bajo una Licencia Creative Commons Atribución-NoComercial-CompartirIgual 3.0 Costa Rica. Para más información envíe un mensaje a 
petrolera entre la cuenca del Volga y los Urales (el principal centro de producción petrolífera se ubicaba en torno a Bakú, Azerbaiyán), la construcción de extensos gasoductos que permitieron el abastecimiento de gas a las ciudades de Moscú, Kiev y Leningrado, además de la inauguración del primer reactor nuclear soviético -y europeo- en la capital soviética (1946) dirigido por el físico atómico Igor Kurchatov (1903-1960), a la que le siguió la primera central atomoeléctrica del mundo instalada en la ciudad científica de Óbninsk (Rusia) en 1954. Cabe señalar la realización de nuevas obras de infraestructura que permitieron la edificación de los proyectos hidroeléctricos rusos en Kuibichev (hoy Samara) y Stalingrado (actual Volgogrado) y la apertura del Canal Volga - Don (1952) que logró comunicar entre los mares de Azov, Báltico, Blanco, Caspio y Negro (Blojintsev y Krasin, 1974 y Lelchuk, Poliakov y Protopopov, 1977).

Los avances experimentados en el sistema de salud soviético permitieron en la década de 1960 la erradicación de enfermedades como la malaria, el cólera, el tifus y la viruela o la drástica disminución de otras como la poliomielitis cuya vacuna fabricada por científicos soviéticos se difundió en otros países por medio de la cooperación internacional (Lelchuk, Poliakov y Protopopov, 1977). Este sistema de salud, de carácter gratuito, era regido por el Ministerio de Salubridad, del que dependían los ministerios de salubridad de cada una de las repúblicas, así como la Academia de Ciencias Médicas y la red de institutos de investigación relacionados con las ciencias de la salud. Además, el gobierno soviético promovió, mediante estímulos económicos y otros de carácter técnico y profesional, la dispersión del personal médico, con el propósito que no se concentrara en las principales ciudades sino que fuera capaz de desarrollar su profesión en las zonas periféricas del país (Cálix, 1971).

La Academia de Ciencias Médicas coordinaba las labores de investigación en el campo de la medicina y llegó a contar con 55 institutos de investigación y 13 
sucursales en todo el país, además se dividió en cuatro secciones (Epidemiología, Microbiología e Higiene; Medicina Clínica, Medico-Biológica y Siberiana) a inicios de la década de 1980 (Mora, 1985).

\section{El estalinismo y las contradicciones en el desarrollo tecnocientífico soviético}

A partir de 1922, cuando Stalin se convierte en el nuevo líder soviético al ser nombrado Secretario General del Comité Central del Partido Comunista de la URSS (PCUS), comienza una etapa de radicalización del proceso revolucionario en la Unión Soviética que se prolongaría hasta el primer lustro de la década de 1950. Ese proceso, conocido bajo el nombre de estalinismo, significó la instauración de un régimen que favoreció el acrecentamiento del poder de un Estado fuertemente centralizado y burocratizado cuyas autoridades ejercían un férreo control del mismo y que se tradujo en una marcada lucha contra la oposición y las voces críticas incluso dentro del partido gobernante (Viales, 2012).

Stalin pretendía lograr la autosuficiencia de la Unión Soviética en el campo científico y tecnológico para sobrepasar a los países capitalistas en estos rubros, resaltando los valores de la ciencia socialista (Ilamada también proletaria), capaz de encontrar aplicaciones útiles a la vida económica y social de los pueblos, de la ciencia capitalista, considerada peyorativamente como "burguesa" (Becerra, 1988).

El fundamento teórico de la actividad científica en la Unión Soviética se basaba en el concepto marxista de materialismo dialéctico, que Stalin definió como "la ideología del partido marxista-leninista [...] porque su procedimiento en el estudio de la Naturaleza, su método de investigar los fenómenos naturales, su método de llegar al conocimiento de estos fenómenos, es el dialéctico, y porque su

La Revista Estudios es editada por la Universidad de Costa Rica y se distribuye bajo una Licencia Creative Commons Atribución-NoComercial-CompartirIgual 3.0 Costa Rica. Para más información envíe un mensaje a 
interpretación de los fenómenos naturales, su concepto de los fenómenos naturales, su teoría, es materialista" (Wetter, 1968, p. 246). La materia fue definida por Lenin como "una categoría filosófica para designar la realidad objetiva, que es dada al hombre en sus sensaciones, que es copiada, fotografiada, reflejada por nuestras sensaciones y que existe independiente de ellas" (Wetter, 1968, p. 29).

Stalin sostenía que el método dialéctico aplicado a la ciencia era capaz de explicar los fenómenos naturales como parte de un todo conformado por la Naturaleza y que dichos fenómenos estaban interrelacionados entre sí (Wetter, 1968, p. 247):

[...] no puede concebirse ni un solo fenómeno en la Naturaleza tomado aisladamente, es decir, sin relación con los fenómenos que lo rodean, pues cualquier fenómeno, y en cualquier terreno del mundo natural, se convertirá en una cosa sin sentido apenas se le considere desvinculado de los fenómenos que lo rodean; al contrario, todo fenómeno puede ser entendido y explicado cuando se le considera dentro de la relación indisoluble y necesaria que lo liga a los fenómenos que lo circundan.

En la década de 1930 con Stalin en el poder, estas ideas se convierten en un dogma para la ciencia soviética y se refuerza con la publicación de su obra Sobre el materialismo dialéctico e histórico (1938), por lo que la investigación científica y tecnológica debían circunscribirse a los postulados ideológicos vigentes y el trabajo de científicos y tecnólogos fuertemente supervisado por el Estado (Wetter, 1968 y Service, 2010). Bajo el régimen estalinista, el apoyo del Estado al desarrollo científico y tecnológico del país fue mayor que el experimentado durante los tiempos de la Nueva Política Económica (NEP), debido a la utilidad que tenían en el campo económico, no obstante, a nivel político, la comunidad científica experimentó también las purgas que tenían lugar en la Unión Soviética, por lo que "muchos investigadores languidecieron en los campos de trabajo de Siberia" (Service, 2010, p. 237).

\section{(c) (†) (-)}

La Revista Estudios es editada por la Universidad de Costa Rica y se distribuye bajo una Licencia Creative Commons Atribución-NoComercial-CompartirIgual 3.0 Costa Rica. Para más información envíe un mensaje a revistaestudios.eeg@ucr.ac.cr. 
De esta forma, la física cuántica comienza a ser cuestionada, en particular algunos de sus aspectos como el principio de incertidumbre de Werner Heisenberg (19011976) ya que la indeterminación era capaz de negar el principio de causalidad y la objetividad inherentes en los fenómenos microfísicos enfocados desde el materialismo dialéctico. Además, se criticaba el intento de aplicar métodos estadísticos relacionados con la medición del comportamiento indeterminado de dichos fenómenos, por lo que se propusieron enfoques teóricos alternativos como las del físico Dmitry Blochinzev (1907-1979) que pudieran conciliar el materialismo dialéctico con la física cuántica, incluso a nivel de mediciones, que excluían la noción de indeterminación (Wetter, 1968).

En cuanto a las teorías de la relatividad propuesta por Albert Einstein (1879-1955), la física soviética consideró inadecuadas algunas interpretaciones catalogadas como simplistas y subjetivas en relación con el materialismo dialéctico. Aunque el matemático Alexander Friedman (1888-1925) llegó a comparar el universo gobernado por las ecuaciones de Einstein - producto de una gran explosión, capaz de colapsar y renacer - con un proceso revolucionario en 1922, se llegó a cuestionar la base filosófica neopositivista de estas teorías y su asociación con la filosofía idealista a la que se le calificó de "burguesa". El físico Serguei Vavilov (1891-1951), quien fue Presidente de la Academia de Ciencias de la URSS, reconoció el carácter revolucionario de las teorías de Einstein pero afirmó que los errores filosóficos que contenía podían subsanarse mediante su adaptación al materialismo dialéctico (Wetter, 1968 y Kojevnikov, 2008).

Wetter (1968, p. 61) expone una de las críticas de la comunidad científica soviética al concepto de curvatura espacial propuesto por la teoría de la relatividad general, a partir de su contenido metafísico:

\section{(c) (i) (2)}

La Revista Estudios es editada por la Universidad de Costa Rica y se distribuye bajo una Licencia Creative Commons Atribución-NoComercial-CompartirIgual 3.0 Costa Rica. Para más información envíe un mensaje a 
Es, a saber: que el espacio del mundo es verdaderamente ilimitado, pero no infinito; es ilimitado algo así como una superficie esférica, pero no infinito. En efecto, de la finitud espacial del universo seguiríase, para los filósofos soviéticos, la limitación y el comienzo del tiempo y, por tanto, la necesidad de una creación del mundo, lo cual va contra el materialismo dialéctico.

En el campo de la cosmología soviética, esta se aferró a la concepción marxista de la infinitud del universo como premisa fundamental para el desarrollo de las investigaciones cosmológicas, por lo que se rechazó la teoría de la muerte térmica del universo, derivada de la Segunda Ley de la Termodinámica planteada por Rudolf Clausius (1822-1888) y propuesta por James Jeans (1877-1946) porque ponía en duda el carácter infinito del universo (Wetter, 1968).

En el plano de la química estructural, se criticó la teoría de la resonancia del estadounidense Linus Pauling (1901-1994), que admitía una diversidad de fórmulas estructurales que eran incapaces de representar en forma fehaciente la propia estructura molecular de un compuesto químico, por lo que fue recibida con escepticismo por parte de los científicos soviéticos, quienes vieron en ella una muestra de idealismo al carecer de una representación objetiva de los compuestos químicos mediante fórmulas estructurales y la asociación que se hizo de esta teoría con la criticada mecánica cuántica (Wetter, 1968).

Otra rama del conocimiento que fue objeto de controversia fue la genética, disciplina que tuvo su origen en los experimentos realizados por el religioso agustino austriaco Gregor Mendel (1822-1884) que desembocaron en el descubrimiento de la transmisión de la herencia biológica de individuos pertenecientes a una generación a sus descendientes. Bajo el estalinismo, la genética se asoció con las prácticas eugenésicas racistas llevadas a cabo en los Estados Unidos durante las primeras décadas del siglo XX y por el régimen nazi en Alemania a partir del decenio de 1930 (Kojevnikov, 2008). 
Es en este contexto que emerge la figura del ingeniero agrónomo Trofim Lisenko (1898-1976), quien planteó un sistema alternativo al de la genética mendeliana, al proponer que la herencia como tal era el resultado de las adaptaciones de los organismos al ambiente donde habitaban. De ahí que el régimen de Stalin apoyó fervientemente al lisenkismo -que se postulaba como ciencia proletaria- a partir de la convocatoria que hizo el Instituto Lenin de Ciencias Agrarias a una asamblea de científicos en Moscú (1948) y lo adoptó como postura científica oficial en las ciencias biológicas, no solo porque servía de contrapeso a la genética mendeliana vigente en Occidente sino porque también las teorías de Lisenko fueron aplicadas al desarrollo de experimentos agrícolas, por ejemplo, su método de vernalización que consistía en someter las sementeras de cereales de invierno a un tratamiento frío para ponerlas a producir en verano y con ello garantizar una mayor productividad (Wetter, 1968 y Kojevnikov, 2008). A pesar del respaldo gubernamental, muchos de sus experimentos fracasaron y sus teorías fueron cuestionadas por la comunidad científica soviética a partir de la década de 1960 (Cheroni, 2004 y Parra, 2013).

Los autores Lelchuk, Poliakov y Protopopov (1977, p. 321) se cuestionaron como el avance en la ciencia y la tecnología soviéticos experimentados a mediados del siglo XX dejaron en segundo plano algunos ramos del conocimiento científico y tecnológico:

Ahora puede parecer extraño que en aquel período no se estimulara el estudio de la cibernética, cesaran casi por completo las investigaciones en algunas ramas de la genética y no se concediera importancia a la introducción de métodos matemáticos en la ciencia económica. Durante los primeros años de postguerra no se prestó el debido apoyo a los trabajos de los investigadores soviéticos en estas esferas, iniciados mucho antes.

\section{(C) $(\Theta \odot$}

La Revista Estudios es editada por la Universidad de Costa Rica y se distribuye bajo una Licencia Creative Commons Atribución-NoComercial-CompartirIgual 3.0 Costa Rica. Para más información envíe un mensaje a 
Aunque los tres autores justificaron esas falencias en el desarrollo tecnocientífico de su país como resultado del proceso mismo de recuperación del impacto de la Segunda Guerra Mundial que condicionó el presupuesto soviético a financiar actividades consideradas prioritarias para el impulso de la economía. Estas prioridades incluyeron la organización de expediciones de reconocimiento a tierras escasamente habitadas, que fueron roturadas para el fomento del desarrollo agrícola y colonizadas por centenares de miles de campesinos en Kazajstán y Siberia; cuya incorporación en la producción de la Unión Soviética fue decisiva para aumentar la producción de cereales a 134 millones de toneladas (1958); además del comienzo de la explotación petrolera y de diamantes en la cuenca del río Lena en la república autónoma de Yakutia (hoy día Sajá) en el oriente de Siberia (Lelchuk, Poliakov y Protopopov, 1977).

El avance en el campo tecnocientífico, significó un desafío para la creciente burocracia soviética, que debió asimilar los cambios generados en la sociedad, tal y como lo expresó el escritor soviético Alexander Bek (1903-1972) en su novela Nuevo nombramiento, escrita en los años sesenta y publicada dos décadas más tarde debido a la censura oficial, donde se cuestionaba la rigidez del sistema administrativo soviético que debía mostrar una actitud más pragmática hacia los avances científicos y tecnológicos para fomentar un crecimiento industrial más eficiente en el país (Popov, 1988). Esta burocratización también se experimentó en la estructura jerárquica de las instituciones científicas soviéticas, donde los intereses políticos e ideológicos prevalecientes condicionaron la producción tecnocientífica, que a su vez fue supeditada a las prioridades requeridas por la planificación central, por lo que algunas ramas del conocimiento terminaron estancándose al no ser consideradas prioritarias para el Estado soviético o tuvieron un desarrollo menor en comparación con los países del bloque occidental (Kara-Murza, 1989).

\section{(c) (i) (2)}

La Revista Estudios es editada por la Universidad de Costa Rica y se distribuye bajo una Licencia Creative Commons Atribución-NoComercial-CompartirIgual 3.0 Costa Rica. Para más información envíe un mensaje a 


\section{La Guerra Fría y sus repercusiones en el desarrollo científico y tecnológico soviético}

Al finalizar la Segunda Guerra Mundial, se constituye un mundo bipolar donde la Unión Soviética lideró el denominado bloque oriental, que incluyó aquellos países donde se instalaron regímenes socialistas afines a Moscú en Europa Central y Oriental. Durante casi medio siglo, el antagonismo entre la Unión Soviética y los Estados Unidos marcó las relaciones internacionales en distintos ámbitos, desde el plano económico hasta el cultural y para garantizar un delicado equilibrio de poder, fue necesario que cada superpotencia contara con el armamento adecuado para contrarrestar las posibles amenazas de su respectivo rival.

Fue precisamente el contexto de la "guerra fría, que impulsó la innovación en determinados campos de la tecnología" (Lewin, 2017, p. 12). Uno de ellos es la energía nuclear, cuyos orígenes se sitúan en la fundación del Instituto Radium de Leningrado (1937) que realiza las primeras investigaciones en este campo, ampliadas posteriormente con los trabajos hechos en el Instituto Kurchatov de Moscú (fundado en 1943) que logró producir la primera reacción atómica controlada en 1946 (De Salazar Serantes, 2004).

En 1949, la Unión Soviética obtiene la bomba atómica y se convirtió en una potencial nuclear para contrarrestar el poderío de los Estados Unidos, a lo que se sumó el desarrollo de la bomba de hidrógeno en 1953. De esta forma, comienza la carrera armamentista entre ambas superpotencias, que llega a su punto máximo tres lustros más tarde con la Crisis de los Misiles en Cuba (1962) que hizo temer a la humanidad por una conflagración nuclear que se evitó gracias a las negociaciones entre los gobiernos soviético y estadounidense que dieron inicio a una etapa de distensión entre ambos superpoderes (Agüero, 2017). De acuerdo con Viales (2012, p. 245), la misma Guerra Fría ejerció una fuerte presión para los

La Revista Estudios es editada por la Universidad de Costa Rica y se distribuye bajo una Licencia Creative Commons Atribución-NoComercial-CompartirIgual 3.0 Costa Rica. Para más información envíe un mensaje a 
países socialistas y en particular la Unión Soviética, porque "alentó un crecimiento y una desviación de fondos hacia la industria bélica", por lo que esta última experimentó una considerable expansión.

Las primeras décadas de la Guerra Fría coincidieron con los procesos de descolonización en África, Asia y América Latina y los nuevos países independientes pasaron a integrar el denominado Tercer Mundo. La Unión Soviética apoyó a los nuevos estados independientes en sus luchas por liberarse de sus metrópolis y logró acuerdos con ellos, especialmente en países donde se instalaron gobiernos socialistas o simpatizantes del modelo soviético (Fazio, 1990). Además de la cooperación económica, el aporte soviético se tradujo en obras de infraestructura para el desarrollo como lo fueron la apertura del complejo metalúrgico de Bhilai (India) y la construcción de la represa de Asuán (Egipto), inaugurada en 1970, que le permitió al país árabe atender muchos problemas derivados del uso de combustibles, producción de energía y labores de riego para la agricultura (Kolontai, 1974 y Ponomariov, Gromyko y Jvostov, 1974).

En el ámbito de la cooperación académica internacional, la Unión Soviética diseñó también un sistema de becas destinado a la formación de científicos, técnicos y personal académico en los países del Tercer Mundo, con el fin de apoyar los procesos de transformaciones sociales y económicas que tenían lugar en varios de esos países (Díaz, 2003). Fruto de esas gestiones fue la fundación de la Universidad para la Amistad de los Pueblos Patrice Lumumba (1960) - Ilamada hoy día Universidad Rusa de la Amistad de los Pueblos y que llevó el nombre del primer ministro del Congo Democrático asesinado en 1961 - donde se formaron profesionales en Ciencias Exactas procedentes de África, América Latina y Asia (Stroganov, 2015). Además, la traducción de obras científicas y técnicas soviéticas a diversas lenguas por parte de la Editorial MIR, fundada en 1946, favoreció la

\section{(c) (i) (2)}

La Revista Estudios es editada por la Universidad de Costa Rica y se distribuye bajo una Licencia Creative Commons Atribución-NoComercial-CompartirIgual 3.0 Costa Rica. Para más información envíe un mensaje a 
difusión del conocimiento tecnocientífico soviético a nivel internacional durante décadas (Zugaza, 2012).

En el marco de la Guerra Fría, se adoptó el Plan Septenal (1959-1965) que aumentó la producción de hulla en la cuenca de Donbáss (oriente de Ucrania) en 80 mil toneladas (1962) mientras que se incrementaba la fabricación de acero en Magnitogorsk y de petróleo en Tartaria y se ponía en operación la central hidroeléctrica de Bratsk en Siberia (1964), en ese entonces la mayor del planeta. No obstante, la aplicación de este mismo plan evidenció el rezago en que había caído el desarrollo agrícola soviético (la agricultura se vio afectada por el severo invierno y el riguroso verano de 1963) y la baja en la producción de bienes de consumo frente al impulso que se le brindó a la industria pesada empezó a dar muestras de agotamiento en cuanto a la aplicación de los procesos de mecanización compleja y automatización (Lelchuk, Poliakov y Protopopov, 1977).

En la década de 1960 se desarrolló la Ciencia de la Información, a la que se le designó con el nombre de Informática en la Unión Soviética, a partir de las investigaciones de Alexander Mijailov (1905-1988), Arkadii Chernyi (1929-2013) y Rudhzero Gilyarevsky (1929) que hicieron posible construir bases de datos de información científica, labor encomendada al Instituto Soviético de Información Científica y Técnica (VINITI) del que Mijailov fue director. Para los investigadores soviéticos, la información científica era más que la recopilación de datos, sino que estos se relacionaban con un contexto social y eran capaces de generar cambios en este, por tanto comprendía toda la "información lógica obtenida en cualquier campo de la actividad humana (no sólo por medio de la investigación, sino también en la actividad práctica y productiva) si ella reflejaba adecuadamente las leyes del mundo objetivo y se empleaba en la práctica histórico-social” (Pedroso, 2004, p. 6).

\section{(c) (i) (2)}

La Revista Estudios es editada por la Universidad de Costa Rica y se distribuye bajo una Licencia Creative Commons Atribución-NoComercial-CompartirIgual 3.0 Costa Rica. Para más información envíe un mensaje a 
El desarrollo de la Informática soviética se encuentra ligada a las políticas impulsadas por Jruschov destinadas a duplicar los logros científicos y tecnológicos procedentes de otros países, especialmente del mundo capitalista y se orientó a reproducir modelos de tecnología que estaba en proceso de desactualización, práctica que tendió a reducirse con la adhesión de la Unión Soviética al Convenio Internacional para la Protección de la Propiedad Industrial (1966) y la Convención Internacional de Derechos de Autor (1973), por lo que estas acciones trajeron consigo un aumento del volumen de la información tecnocientífica generada en el extranjero (Becerra, 1988).

Otro aspecto de la Guerra Fría que incentivó la ciencia y la tecnología soviética fue la carrera espacial, que también fue un ejemplo de la relación que se estableció entra la producción científica y el desarrollo industrial del país. De acuerdo con Mijail Millionschikov (1974, p. 25), quien fue Vicepresidente de la Academia de Ciencias de la URSS: "La alianza de la ciencia soviética con la industria ha hallado su admirable encarnación en los éxitos de la cohetería y en una de las mayores realizaciones de la época: la penetración del hombre en los espacios cósmicos".

En 1955 abrió sus puertas el cosmódromo de Baikonur (Kazajstán) desde donde se lanzó el primer satélite artificial Sputnik I (1957) que permitió la comprobación de las leyes planteadas por Johannes Kepler (1571-1630) en el siglo XVII y al primer ser viviente fuera de la atmósfera: la perra Laika, enviada al espacio en el Sputnik // ese mismo año. La Unión Soviética tiene el mérito de haber colocado al primer hombre en el espacio, Yuri Garin (1934-1968), en 1961 y a la primera mujer, Valentina Tereshkova (1937) en 1963 y fue un cosmonauta soviético, Alexei Leonov (1934), quien efectuó la primera caminata espacial el 18 de marzo de 1965 (Lelchuk, Poliakov y Protopopov, 1977 y Paluzíe, 1984). Además, en esta carrera espacial, también hubo fracasos, como la muerte del cosmonauta Vladimir Komarov (1927-1967), quien fue el primer ser humano en morir en un accidente

La Revista Estudios es editada por la Universidad de Costa Rica y se distribuye bajo una Licencia Creative Commons Atribución-NoComercial-Compartirlgual 3.0 Costa Rica. Para más información envíe un mensaje a 
Revista Estudios, (36), 2018.

Junio 2018-Noviembre 2018

ISSN 1659-3316

Díaz Bolaños Ronald Eduardo

relacionado con la exploración al espacio cuando la Soyuz 1 se estrelló al regresar de su misión (Klimuk, 1986).

El establecimiento de la Ciudad de las Estrellas, en las proximidades de Moscú, fue fundamental en la formación de los cosmonautas soviéticos y de otros países socialistas; además de ser un importante centro científico, tuvo también una función cultural y de socialización (Klimuk, 1986, pp. 169 y 197):

La Casa de la Cultura de la Ciudad Estelar efectúa una gran labor ideo-estética. Allí funcionan universidades de marxismo-leninismo y de cultura, diversos lectorios y escuelas [...] incorporar activamente a los cosmonautas de la Ciudad Estelar a las actividades sociales y de masas, a la labor cultural y estética [...]. La Ciudad Estelar se ha convertido en un centro internacional de preparación de cosmonautas. Nuestros compañeros ayudaron a cosmonautas de los países socialistas a dominar en corto plazo el vasto y complicado programa de preparación para los vuelos. Se hizo todo lo posible para realizar eficazmente con ellos cada ejercicio, cada experimento.

De esta forma pudieron incorporarse los cosmonautas procedentes del bloque oriental (Alemania Oriental, Bulgaria, Checoslovaquia, Hungría, Polonia y Rumania) y de otros países socialistas (Cuba, Mongolia, Vietnam) y otros países como India y Francia (Pashuto et al., 1985).

El programa espacial soviético se orientó a la exploración de varios cuerpos del sistema solar como lo fueron la Luna, Marte y Venus, a través del lanzamiento de las sondas Lunik, Mars y Venera, respetivamente, que fueron enviadas a dichos astros entre finales de la década de 1950 y mediados de la de 1980 y entre sus logros se destaca la toma de las primeras fotografías de la cara de la Luna que no es visible desde la Tierra y la transmisión televisada de un alunizaje (1965), además de la colocación del Lunojod-1, primer robot que exploró la superficie

\section{(c) (i) (2)}

La Revista Estudios es editada por la Universidad de Costa Rica y se distribuye bajo una Licencia Creative Commons Atribución-NoComercial-CompartirIgual 3.0 Costa Rica. Para más información envíe un mensaje a revistaestudios.eeg@ucr.ac.cr. 
lunar y transmitió datos hacia la Tierra (1970). La cooperación entre países socialistas se hizo presente también en el lanzamiento del satélite Intercosmos-1 (1969), en cuyo planeamiento participó personal científico procedente de Rumania, Polonia, Hungría y Bulgaria; fue equipado con tecnología soviética, germanooriental, china y se emplearon componentes fabricados en Francia (Lelchuk, Poliakov y Protopopov, 1977 y Paluzíe, 1984).

La Unión Soviética fue también pionera en la colocación de estaciones espaciales por medio del proyecto Salyut, cuya primera versión fue lanzada al espacio en 1971 y la última en 1982, las cuales recibieron misiones tripuladas mediante el acoplamiento de las cosmonaves Soyuz, que permitieron la realización de experimentos, entre ellos el estudio del comportamiento humano en condiciones de ingravidez (Paluzíe, 1984 y Millán, 2000).

La distensión que reinaba entre ambas superpotencias llegó también al espacio con la Misión Soyuz-Apolo (1975), organizada por las agencias espaciales soviética y estadounidense. El Soyuz XIX despegó de Baikonur con los cosmonautas Alexei Leonov y Valery Kubásov (1935-2014) y el Apolo fue lanzado desde el Centro Espacial Kennedy en Cabo Cañaveral (Estados Unidos) con los astronautas Vance Brand (1931), Donald Slayton (1924-1993) y Thomas Stafford (1930), quienes conformaron un equipo internacional de investigación que efectuó experimentos conjuntos en el campo de la astrofísica, astronomía, biología e investigación aplicada al campo industrial. Ambas naves permanecieron acopladas durante casi dos días orbitando al planeta (Paluzie, 1984).

Mientras los avances soviéticos de su programa espacial asombraban a la humanidad, la llegada al poder de Leonid Brezhnev (1906-1982) en 1964 favoreció el replanteamiento de la planificación central con miras a fortalecerla y se efectuaron algunas reformas para el impulso de la agricultura y la industria

\section{(c) (i) (2)}

La Revista Estudios es editada por la Universidad de Costa Rica y se distribuye bajo una Licencia Creative Commons Atribución-NoComercial-CompartirIgual 3.0 Costa Rica. Para más información envíe un mensaje a 
(incluyendo la electrónica y la radiotecnia) en el marco del denominado "socialismo desarrollado" (Lelchuk, Poliakov y Protopopov, 1977 y Service, 2010). No obstante, estas no supusieron cambios drásticos en la política económica soviética que ya en la década de 1970 comenzaba a dar muestras de agotamiento del modelo económico socialista debido a la desproporción existente en el crecimiento de la industria pesada con respecto a la manufactura de bienes de consumo y a la agricultura. Además, la industria soviética no fue capaz de adaptarse a las nuevas tecnologías y resintió la fuerte inversión que se hacía en la industria militar; la Primera Crisis del Petróleo (1973-1974) que hizo aumentar las exportaciones de petróleo soviético permitió una mejora en las condiciones de la economía del país sin que se tradujera en reformas sustanciales y se destinaron más recursos para financiar la carrera armamentista (Hobsbawm, 1996, Díaz, 2012 y Agüero, 2016).

Aun así Brezhnev, en el marco de la distención entre ambas superpotencias, se mostró favorable a la cooperación en el ámbito de la ciencia y la tecnología, siguió invirtiendo en el desarrollo de la industria pesada, promoviendo las prácticas de duplicación y la adquisición de tecnología extranjera que resultaba más económica que producirla en la misma Unión Soviética (Becerra, 1988). Eso fue lo que sucedió con el desarrollo de la informática y la computación en la sociedad soviética: Serguei Lebedev (1902-1974), Director del Instituto Electrotécnico de Kiev - perteneciente a la Academia de Ciencias de Ucrania - fabricó el primer prototipo de computadora en la Unión Soviética en 1948 y cinco años más tarde, se instaló en Moscú la primera gran computadora BESM-1 que poseía 180 mil transmisores individuales y carecía de microcircuitos. Sin embargo, el desarrollo de la computación estuvo limitado debido a sus vínculos con la cibernética, campo que fue catalogado como "pseudociencia burguesa" por las autoridades soviéticas al atribuirle la pretensión de reproducir las leyes de la Naturaleza en los sistemas tecnológicos. No obstante, en la década de 1960, se introdujeron computadoras personales de la serie Mir-1 a la Mir-3 que carecían de un sistema operativo

\section{(c) (i) (2)}

La Revista Estudios es editada por la Universidad de Costa Rica y se distribuye bajo una Licencia Creative Commons Atribución-NoComercial-CompartirIgual 3.0 Costa Rica. Para más información envíe un mensaje a 
unificado, falencia que trató de resolverse mediante la reproducción del computador IBM/360 en 1969 lo que provocó el estancamiento de la producción de computadoras soviéticas por la ausencia de innovaciones que permitieran avances en este sector tecnológico (Ter-Gazarian, 2014 y Valdés y Valdés, 2014).

Bajo el mandato de Brezhnev se visibiliza aún más el descontento de sectores de la intelectualidad soviética - intelligentsia - en general y de la comunidad científica en particular, con el régimen gobernado por el PCUS, siendo el físico nuclear Andrei Sajarov (1921-1989) el más célebre científico disidente. Sajarov recibió el Premio Nobel de la Paz en 1975 después de haber cuestionado la falta de libertad de expresión en la Unión Soviética después de haberse iniciado el proceso de desestalinización, había colaborado con la fundación de un Comité de Derechos Humanos (1970) y planteó una sociedad democrática como el espacio idóneo para la práctica de los derechos civiles. El Estado soviético, como represalia por sus ideas, le obligó a residir en la ciudad rusa de Gorki (actual Nizhni Nóvgorod) a partir de 1980 hasta que fue rehabilitado años más tarde por Gorbachov (Service, 2010).

Sajarov fue parte de una generación de intelectuales disidentes a la que también pertenecieron los escritores Alexander Solzhenitsyn (1918-2008) y Alexander Tvardovsky (1910-1971), editor de la revista Novyi Mir; además del historiador Roy Medvedev (1925), quienes mantuvieron una actitud crítica hacia el régimen de Moscú y abogaban por reformas políticas en el país, que se hicieron realidad hasta el decenio de 1980 (Service, 2010 y Lewin, 2017).

\section{(c) (i) (2)}

La Revista Estudios es editada por la Universidad de Costa Rica y se distribuye bajo una Licencia Creative Commons Atribución-NoComercial-Compartirlgual 3.0 Costa Rica. Para más información envíe un mensaje a 


\section{La ciencia y la tecnología en la etapa final de la Unión Soviética}

Hacia 1980, mientras Moscú celebraba los XXII Juegos Olímpicos de la Era Moderna y las tropas soviéticas combatían a los muyahidines en Afganistán desde hacía un año, los cuadros científicos de la superpontencia conformaban alrededor de un cuarto del total de la comunidad científica del planeta (Efimov, 1982). Un año más tarde, con la llegada al poder de Ronald Reagan (1911-2004) en los Estados Unidos, con su férreo discurso belicista antisoviético, se produjo el rebrote de la Guerra Fría, lo que provocó una disminución en el intercambio entre las instituciones científicas soviéticas y estadounidenses iniciada años atrás durante la etapa de distensión. Esta coyuntura coincidió con el final del mandato de Brezhnev y lo breves períodos de Yuri Andropov (1914-1984) y Konstantin Chernenko (1911-1985) como Secretarios Generales del PCUS entre 1982 y 1985 (Mora, 1985).

En este contexto, la filial de la Academia de Ciencias de la URSS en Novosibirsk, ciudad industrial situada a más de 3 mil kilómetros al este de Moscú, contó con su propia Ciudad Académica y estaba integrada por 23 institutos de investigación científica a inicios de la década de 1980, gozaba de un ambiente de mayores libertades para la producción científica, en particular de los "investigadores de vanguardia", quienes se trasladaban a ella debido a los menores controles ejercidos por el aparato estatal hacia el personal científico (Mora, 1985 y Carrère d'Encausse, 2016).

De acuerdo con un informe del Departamento de Defensa de los Estados Unidos (1983, p. 76), la Unión Soviética había logrado conformar una importante infraestructura en el campo militar que le permitía preservar su seguridad al iniciar la década de 1980:

\section{(C) $(\Theta \odot$}

La Revista Estudios es editada por la Universidad de Costa Rica y se distribuye bajo una Licencia Creative Commons Atribución-NoComercial-CompartirIgual 3.0 Costa Rica. Para más información envíe un mensaje a 
Para el sostenimiento de tan amplio crecimiento militar, los soviéticos tienen un sistema centralizado y bien organizado que abarca a un núcleo de nueve ministerios industriales muy implicados en los programas militares, el llamado 'Sector Industrial Soviético de Defensa'. Bajo la dirección de la Defensa, estos ministerios proyectan las armas, desarrollan prototipos y, tras pasar las pruebas de evaluación del ministerio de Defensa, producen las armas.

El mismo informe estimaba que en 1982 había más de 900 mil científicos e ingenieros trabajando en la industria militar soviética mientras en los Estados Unidos sus pares no llegaban a los 700 mil, cifra que correspondía al 50\% o 75\% de todo el personal tecnocientífico soviético, en una época en que la infraestructura militar soviética había crecido de tal forma que su tecnología militar se equiparaba con la estadounidense. La inversión en la carrera armamentista por parte de la Unión Soviética correspondía a un 15\% de su Producto Nacional Bruto (PNB) (Departamento de Defensa de los Estados Unidos, 1983).

El Departamento de Defensa de los Estados Unidos (1983, p. 78) advirtió el impacto que tenía el fuerte gasto militar para la estabilidad económica de la Unión Soviética, lo que también se manifestó en otros países socialistas europeos:

La Unión Soviética y los países del Pacto de Varsovia han afrontado en el pasado decenio un rendimiento económico descendente, mientras que mantenían niveles altos de producción de material de guerra para una modernización acelerada de sus fuerzas. La economía soviética está acosada por una creciente escasez de recursos, mayores costes de producción y competencias de prioridades entre sectores. Las penurias alimenticias, baja productividad laboral y la desorganización de los transportes han coincidido para llevar el crecimiento industrial a niveles comparables a los inmediatamente posteriores a 1945.

En 1985, con la llegada de Mijail Gorbachov (1931) al poder, se comprende la necesidad de reformar el sistema soviético con el fin de sacar del estancamiento a 
la URSS y de paso emprender una política tendiente a borrar la huella del estalinismo superviviente a través de dos objetivos fundamentales: "El primer objetivo era modernizar la economía soviética y su estructura política y social. Este proceso fue llamado la Perestroika. Además, se propuso asegurar la libertad de la información y el ejercicio del libre pensamiento. A este segundo objetivo se le denominó la Glasnot (Díaz, 2012, p. 114 [el texto en negrita procede del original]).

Frente al desafío que significaba el agotamiento del modelo socialista soviético, la política tecnocientífica de la etapa final de la Unión Soviética fue condicionada por la compleja situación económica experimentada en el decenio de 1980 que llevó a un rezago tecnológico en la producción de bienes de consumo y la inercia en el crecimiento de una industria que carecía de competitividad a nivel internacional, de ahí que se pensó en la apertura a Occidente con el fin de estimular el desarrollo científico y tecnológico mediante la cooperación internacional y reducir el control estatal en el rubro de la ciencia y la tecnología (Becerra, 1988).

El mismo Gorbachov abogó por una especie de Perestroika en el campo científico y tecnológico, orientada a la disminución del peso de la burocracia y los controles políticos e ideológicos que condicionaron las investigaciones o repercutieron en el estancamiento de algunas ramas del ámbito tecnocientífico soviético, incluso la posibilidad de reestructurar la Academia de Ciencias de la URSS y replantear su papel en la esfera productiva del país (Kara-Murza, 1989). Además, se trató de reforzar las relaciones económicas con los países emergentes del Tercer Mundo con el propósito de establecer un mayor intercambio en el ámbito tecnológico (Fazio, 1990).

A pesar de las dificultades experimentadas por el desarrollo tecnocientífico en este contexto, tuvo lugar un nuevo hito en la carrera espacial con la colocación en órbita de la estación espacial Mir en 1986 -en coincidencia con la celebración del 
XXVII Congreso del PCUS - y se mantuvo en operaciones durante quince años sobreviviendo al colapso de la URSS, con el fin de realizar investigaciones previas a la introducción de actividades productivas en el espacio. La primera tripulación estuvo conformada por los cosmonautas Leonid Kizim (1941-2010) y Vladimir Solovyov (1946), quienes permanecieron en el espacio durante 125 días. A la estación espacial se le fueron acoplando una serie de módulos en los siguientes tres años pero los cambios económicos y políticos que experimentaba la URSS hicieron que el programa de transbordadores espaciales soviéticos Burán - que debían enviarse al espacio para acoplarse con la Mir - se suspendiera, no obstante, se pudo batir el récord de permanencia humana en el espacio cuando los cosmonautas Vladimir Titov (1947) y Musa Manárov (1951), procedente de Azerbaiyán, permanecieron 366 días en el espacio entre 1987 y 1988. El último cosmonauta que representó a la Unión Soviética fue Alexander Volkov (1948), quien arribó a la Mir en 1991 y regresó a tierra junto con su compañero Serguei Krikaliov (1958), convertidos en ciudadanos rusos (Millán, 2000 y Marín, 2016).

\section{Impactos ambientales de algunos proyectos tecnocientíficos soviéticos}

En 1946, una fuerte sequía afectó las zonas rurales de Crimea, Ucrania, Moldavia y la cuenca del Volga, que provocó el decrecimiento en la producción de cereales, mientras el país se recuperaba de los efectos de la Gran Guerra Patria, por lo que el Estado intervino y empleó sus reservas y prolongó el racionamiento de productos alimenticios para superar la crisis provocadas por la carestía. Dos años más tarde, en 1948, un violento terremoto destruyó Asjabad, capital de Turkmenistán, por lo que fue necesaria la ayuda solidaria de otras repúblicas soviéticas para atender a los damnificados y reparar los cuantiosos daños producidos en la infraestructura de la ciudad turcomana (Lelchuk, Poliakov y Protopopov, 1977).

\section{(C) $(00$}

La Revista Estudios es editada por la Universidad de Costa Rica y se distribuye bajo una Licencia Creative Commons Atribución-NoComercial-CompartirIgual 3.0 Costa Rica. Para más información envíe un mensaje a 
No obstante, la respuesta a los desafíos ambientales en relación con el desarrollo económicos fueron las adecuadas, siendo uno de los más significativos el programa de irrigación de los campos de algodón cultivados a gran escala en las repúblicas centroasiáticas mediante el desvío del agua de los ríos Amú Daria y Sir Daria, que abastecían al Mar de Aral (ubicado entre Uzbekistán y Kazajstán). Si bien es cierto, los proyectos de regadío y cultivo de algodón de esta zona databan del período zarista, bajo la Unión Soviética se expandió esta actividad durante la segunda mitad del siglo $\mathrm{XX}$ con la puesta en marcha del proyecto de Tierras Vírgenes auspiciado por el mismo Jruschov. Este proyecto permitió que en Uzbekistán las tierras dedicadas a la producción algodonera pasaran de 1022600 hectáreas en 1940 a 2103300 en 1987 (De La Iglesia, 2006).

De acuerdo con De La Iglesia (2006, p. 504), la expansión agrícola algodonera tuvo un fuerte impacto en las condiciones económicas, sociales y ambientales en las costas del Mar de Aral debido a los drásticos cambios que generó:

En 1960 el Aral era el cuarto mar interior del mundo. Sus playas eran frecuentadas por miles de turistas procedentes de otras repúblicas de la URSS, y en la desembocadura del Amú Daria y del Syr Daria reinaba una diversidad de flora y fauna bien apreciada más allá de las fronteras regionales [...] En la década de 1960 había en el Aral una floreciente industria pesquera basada en unas capturas anuales cifradas en 40.000 toneladas; industria en la que trabajaban más de 60.000 personas en los diferentes procesos que iban desde la pesca a la selección, conservación, envasado y transporte de extraídos de sus fértiles aguas, aún no contaminadas por los millones de toneladas de productos químicos que fueron vertidas más tarde en las tierras de regadío dedicadas al cultivo del algodón.

La ampliación del sistema de regadío provocó que en las décadas siguientes los dos grandes ríos anteriormente mencionados surtieran menos agua al Mar de Aral 
y por consiguiente su nivel descendió, su superficie disminuyó drásticamente dejando tras de sí acumulación de sal en tierras ganadas por el desierto que provocaron la separación de este mar interior en dos secciones (Mar de Aral Norte y Sur). La actividad pesquera y turística declinaron provocando un enorme perjuicio económico a la población de sus antiguas riberas, quienes abandonaron sus embarcaciones en medio de la superficie desértica, además de la pérdida provocada en los ecosistemas lacustres (De La Iglesia, 2006). A inicios del siglo $X X I$, se han realizado esfuerzos por rescatar lo que queda del Mar de Aral mediante el planeamiento y construcción de obras hidráulicas, la recuperación de las especies de peces comestibles para reintroducir la actividad pesquera, especialmente en el Mar de Aral Norte y el uso de variedades alternativas de algodón que requieren un menor aprovechamiento de agua que las tradicionales (López, 2016 y Reuters, 2017).

Otro proyecto que ha tenido importantes implicaciones ambientales ha sido el Polígono de Semipalatinsk (Kazajstán), abierto en 1949 como campo de pruebas nucleares - se efectuaron 470 durante cuatro décadas - que llegó a contaminar un área de $300 \mathrm{mil} \mathrm{km}^{2}$, debido a la difusión de polvo radiactivo generado por las detonaciones y afectó alrededor de medio millón de personas, de las que más de la quinta parte falleció por enfermedades cancerígenas (De La Iglesia, 2006).

El 26 de abril de 1986 tuvo lugar la explosión de uno de los cuatro reactores de grafito en la central nuclear de Chernóbil (Ucrania), cuyos efectos inmediatos se hicieron sentir en zonas del occidente de la Unión Soviética, en tierras bielorrusas, rusas y ucranianas, pobladas por unos cinco millones de habitantes y que provocó la reubicación de las poblaciones aledañas que sufrieron los impactos directos de la radiación, ya que el material radiactivo equivalía a más de cien veces el que fue liberado por ambas bombas atómicas lanzadas en Japón (1945). El accidente

\section{(c) (i) (2)}

La Revista Estudios es editada por la Universidad de Costa Rica y se distribuye bajo una Licencia Creative Commons Atribución-NoComercial-Compartirlgual 3.0 Costa Rica. Para más información envíe un mensaje a 
Revista Estudios, (36), 2018.

Junio 2018-Noviembre 2018

ISSN 1659-3316

Díaz Bolaños Ronald Eduardo

también redujo en una sétima parte la producción de electricidad generada por los reactores de esta planta (Agüero, 2016).

El accidente de Chernóbil tuvo profundas repercusiones a nivel político, en particular cuando sus efectos se hicieron sentir más allá de las fronteras soviéticas y ya no fue posible ocultar el impacto social del suceso al interior del país. De acuerdo con Carrère d'Encausse $(2016,44)$ :

Las noticias que llegaron entonces fueron más precisas, más trágicas; sin embargo, no cesó la discreción del Kremlin. Durante más de tres semanas, el poder soviético impuso un silencio casi total al país, mientras que en los días que siguieron a la explosión, una nube radiactiva alcanzó Suecia y después avanzó hacia Europa occidental [...] La política de espera del Kremlin tuvo consecuencias dramáticas. En primer lugar, en el mismo sitio, en torno a la central. Las evacuaciones, ya de entrada, fueron insuficientes. A partir de las primeras noticias que se recibieron, el Politburó sacó la conclusión de que el peligro se limitaba a Ucrania, pero los vientos orientaron la contaminación radiactiva hacia Bielorrusia, cuya población pagó un tributo espantoso a ese error de apreciación. Hubo que esperar hasta el 14 de mayo, casi tres semanas después de la catástrofe, para que Gorbachov se dirigiera a su país en una alocución televisada y reconociera, sin cautela esta vez, lo que había pasado en Chernóbil.

Incluso, como lo señala Agüero (2017, p. 3), el accidente en Chernóbil contribuyó a cambiar la imagen que tenía la población afectada del progreso científico al concebirse ahora como "una ciencia repudiada, luego de que fuera promocionada como portadora de grandes ventajas, merced a la utilización de la energía atómica para la paz" teniendo en cuenta que las autoridades soviéticas abogaron por el uso pacífico de este tipo de energía durante la Guerra Fría, incluso la Academia de Ciencias Médicas propugnó la lucha contra la guerra nuclear mediante la fundación de la organización "Médicos para la Prevención de la Guerra Nuclear"

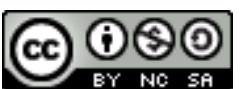

La Revista Estudios es editada por la Universidad de Costa Rica y se distribuye bajo una Licencia Creative Commons Atribución-NoComercial-CompartirIgual 3.0 Costa Rica. Para más información envíe un mensaje a revistaestudios.eeg@ucr.ac.cr. 
Revista Estudios, (36), 2018.

Junio 2018-Noviembre 2018

ISSN 1659-3316

Díaz Bolaños Ronald Eduardo

(Mora, 1985 y Pashuto et al., 1985). Ya en 1957, había ocurrido un accidente en la planta nuclear Cheliábinsk-40, ubicada cerca de Kyshtym (Rusia) y cuyos efectos no se conocieron hasta 1989 (Agüero, 2016).

\section{Conclusiones}

La Unión Soviética, como superpotencia del bloque socialista surgido al finalizar la Segunda Guerra Mundial, se destacó en el campo de la ciencia y la tecnología, lo que se evidenció en el desarrollo de la carrera armamentista y en la exploración espacial a lo largo del período de la Guerra Fría. Todo este desarrollo es el resultado de una política científica impulsado por el Estado soviético desde sus inicios, en el que se incorporó al personal científico de la Rusia zarista al proyecto socialista de Lenin, que hizo posible la transformación de una economía preponderantemente agrícola a una de carácter industrial, especialmente a partir de la aplicación de la NEP y los primeros planes quinquenales en tiempos de Stalin. Además, el sistema educativo soviético favoreció la introducción de los cursos de ciencia en el currículo de los estudiantes, lo que sin duda incentivó el interés por las investigaciones científicas y tecnológicas en el país.

De esta forma, la ciencia y la tecnología colaboraron con la construcción del modelo económico soviético, debido al carácter práctico que adquirieron sus investigaciones, con el fin de brindar respuesta a los problemas sociales y económicos generados por la implantación del sistema socialista en el país más extenso de la Tierra. En este proceso fue fundamental el papel desempeñado por la Academia de Ciencias de la Unión Soviética, que se convirtió en la institución ejecutiva de la política científica y tecnológica desarrollada en la superpotencia y para ello contó con una estructura jerarquizada conformada por las academias de las repúblicas federadas y las regionales, además de una serie de institutos

\section{(C) $(00$}

La Revista Estudios es editada por la Universidad de Costa Rica y se distribuye bajo una Licencia Creative Commons Atribución-NoComercial-CompartirIgual 3.0 Costa Rica. Para más información envíe un mensaje a revistaestudios.eeg@ucr.ac.cr. 
científicos y centros de investigación distribuidos a lo largo y ancho de su vasto territorio.

Por un lado, la centralización del desarrollo tecnocientífico por parte del Estado soviético le permitió contar con fondos para la realización de las investigaciones de las instituciones científicas pero por otro, condicionó sus objetivos al supeditarse a las exigencias de la planificación central. Otra limitación fue que la ciencia y la tecnología soviéticas estuvieron fuertemente influenciadas por el dogmatismo ideológico en tiempos de Stalin que canonizó al materialismo dialéctico como fundamento para toda investigación científica y tecnológica, lo que limitó el campo de acción de algunas ramas del conocimiento, mientras que los desarrollos teóricos procedentes del mundo occidental debieron ser interpretados desde una óptica marxista para su aceptación en el ámbito científico soviético o bien, su sustitución por teorías más acordes con los postulados ideológicos del marxismo-leninismo.

La ciencia y la tecnología soviética también reflejaron los cambios experimentados a través del tiempo por la sociedad que los produjo, ya que en ella se evidenció el proceso de desestalinización que favoreció el desarrollo de algunas áreas del conocimiento que habían sido más limitadas o cuestionadas bajo el régimen estalinista. Además, la crisis del sistema soviético se hizo presente en el campo tecnocientífico, debido al empeño que se dio a la industria pesada, militar y aeroespacial frente a otra destinada a la producción de bienes de consumo. Las reformas de Gorbachov también pretendieron dinamizar el ámbito científico y tecnológico y replantear su papel en una nueva Unión Soviética, sin embargo, su disolución llevó a que cada una de las repúblicas heredara el acervo científico y tecnológico desarrollado en ellas durante el período soviético y la Academia de Ciencias de la URSS pasó a llamarse de nuevo Academia de Ciencias de Rusia

\section{(c) (i) (2)}

La Revista Estudios es editada por la Universidad de Costa Rica y se distribuye bajo una Licencia Creative Commons Atribución-NoComercial-CompartirIgual 3.0 Costa Rica. Para más información envíe un mensaje a 
en 1991, al quedar una parte significativa de su infraestructura en la Federación Rusa.

No obstante, algunos proyecto tecnocientíficos que se desarrollaron en territorio soviético tuvieron un impacto ambiental de grandes repercusiones (en algunos clases internacionales), como el accidente de Chernóbil, la contaminación generada por el campo de pruebas de Semipalatinsk y la desecación del Mar de Aral, cuyos efectos siguen manifestándose en el presente.

Finalmente, cabe señalar el apoyo que brindó la desaparecida Unión Soviética en el financiamiento de algunos proyectos como la represa de Asuán en Egipto y la conformación de cuadros tecnocientíficos en los países en vías de desarrollo, que tuvo lugar en forma paralela a la Guerra Fría, por lo que debe estudiarse también su papel en el desarrollo científico y tecnológico en países como Costa Rica.

\section{Bibliografía}

Agüero, J. (2016). El desmoronamiento del mundo socialista en Europa. San José: EUCR.

Agüero, J. (2017). La plegaria de Chernóbil: memoria del desastre nuclear en el contexto de un poder agonizante. En Revista Estudios 35, pp. 1-49.

Armytage, W.H.G. (1962) The origins of Russian scientific and technological training. In The Vocational Aspect of Secondary and Further Education, 14 (29), pp. 79-91, DOI: 10.1080/03057876280000081.

\section{(c) (1) (2)}

La Revista Estudios es editada por la Universidad de Costa Rica y se distribuye bajo una Licencia Creative Commons Atribución-NoComercial-Compartirlgual 3.0 Costa Rica. Para más información envíe un mensaje a 
Balashova, Y. B. (2016). The Scientific Enlightenment System in Russia in the Early Twentieth Century as a Model for Popularizing Science. In International Journal of Enviromental \& Science Education 11 (18), pp. 11467-11476.

Becerra, M. (1988). "El derecho de invención y la ciencia y tecnología en la URSS. En Cuadernos del Instituto de Investigaciones Jurídicas, pp. 739-755.

Black, C. E. (1969). Naturaleza de la sociedad imperial rusa. En Treadgold, D. W., dir. El desarrollo de la U.R.S.S. Estudios y debates. Madrid: Tecnos, pp. 201-219.

Blojintsev, D. I. y Krasin, A. K. (1974). Construcción y explotación de la primera central nuclear. Evocación de algunos problemas y soluciones, pp. 1-12. Recuperado de https://www.iaea.org/sites/default/files/16304700712_es.pdf.

Cálix, R. F. (1971). La Medicina Socializada. En Revista Médica Hondureña 39 (1), pp. 27-32.

Carrère d'Encausse, H. (2016). Seis años que cambiaron el mundo. 1985-1991, la caída del imperio soviético. Barcelona: Ariel.

Cheroni, A. (2004). El caso Lisenko: Una relectura. En Llull, 27 (60), pp. 609-630.

De La Iglesia, J. (2006). El desastre ecológico de Asia Central. En Anuario Jurídico y Económico Escurialense XXXIX, pp. 493-522.

De Salazar Serantes, G. (2004). El sector nuclear exsoviético. En Documentos CIDOB, Seguridad y Defensa 4, pp. 122-163. Recuperado de https://www.cidob.org/content/download/2830/24799/version/2/file/SALAZARcapitu lo3.pdf.

\section{(c) (i) (2) (2)}

La Revista Estudios es editada por la Universidad de Costa Rica y se distribuye bajo una Licencia Creative Commons Atribución-NoComercial-CompartirIgual 3.0 Costa Rica. Para más información envíe un mensaje a revistaestudios.eeg@ucr.ac.cr. 
Departamento de Defensa de los Estados Unidos de América (1983). El poderío militar soviético. Madrid: Editorial San Martín.

Díaz, D. (2012). Estado, sociedades y nacionalismo en un mundo globalizado. En Díaz, D.; Viales, R. y Marín, J. J. Historia de la Cultura. San José: EUNED, pp. 1148.

Díaz, R. (2003). El proceso de institucionalización de la Meteorología en Costa Rica. Tesis de Licenciatura en Historia. Universidad de Costa Rica.

Efimov, N. (1982). El modo de vida soviético. Moscú: Editorial de la Agencia de Prensa Nóvosti.

Fazio, H. (1990). La Unión Soviética y el Tercer Mundo. En Historia Crítica 3, pp. 5-19.

Hessen, B. (1989). Las raíces socioeconómicas de la mecánica de Newton. En Saldaña, J. J. (comp.). Introducción a la teoría de la historia de las ciencias. México: UNAM, pp. 79-145.

Hobsbawm, E. (1996). Historia del siglo XX. Barcelona: Crítica.

Kara-Murza, S. (1989). Ciencia y burocratismo: Experiencia soviética. En Llull 12 (23), pp. 397-414.

Keldych, M. (1970). Lenin y el desarrollo de la ciencia. En El Correo de la UNESCO XXIII, julio, pp. 6-11.

\section{(c) (i) (2)}

La Revista Estudios es editada por la Universidad de Costa Rica y se distribuye bajo una Licencia Creative Commons Atribución-NoComercial-CompartirIgual 3.0 Costa Rica. Para más información envíe un mensaje a revistaestudios.eeg@ucr.ac.cr. 
Revista Estudios, (36), 2018.

Junio 2018-Noviembre 2018

ISSN 1659-3316

Díaz Bolaños Ronald Eduardo

Klimuk, P. (1986). Tras el horizonte del universo. En Mitroshénkov, V. A., comp. El

programa espacial soviético: páginas de historia. Moscú: Progreso, pp. 158-198.

Kojevnikov, A. (2008). The Phenomenon of Soviet Science. In OSIRIS 23, pp. 115135.

Kolontai, V. (1974). La Revolución tecnocientífica y los países en desarrollo. En Academia de Ciencias de la Unión Soviética. La Revolución tecnocientífica: Aspectos y perspectivas sociales. Moscú: Progreso, pp. 232-251.

Lelchuk, V.; Polyakov, Y y Protopopov, A. (1977). Historia de la sociedad soviética. Moscú: Progreso.

Leonov, V. P., ed. (2014). 300 th anniversary of the Russian Academy of Sciences Library. Moscow: Russian Academy of Sciences Library. Recovered from http://www.rasl.ru/e editions/300ban buklet.pdf.

Lewin, M. (2017). El siglo soviético. ¿Qué sucedió realmente en la Unión Soviética? Barcelona: Crítica.

López, M. A. (2016, 8 mayo). La historia más triste de todos los mares: Aral. En Geografía infinita. Recuperado de https://www.geografiainfinita.com/2016/05/lahistoria-mas-triste-de-todos-los-mares-aral/.

Marín, D. (2016, 22 febrero). 30 años de la Mir, la primera estación espacial internacional. En Eureka. Recuperado de http://danielmarin.naukas.com/2016/02/22/30-anos-de-la-mir-la-primera-estacionespacial-internacional/.

\section{(c) (i) (2)}

La Revista Estudios es editada por la Universidad de Costa Rica y se distribuye bajo una Licencia Creative Commons Atribución-NoComercial-CompartirIgual 3.0 Costa Rica. Para más información envíe un mensaje a revistaestudios.eeg@ucr.ac.cr. 
Millán, G. (2000). La conquista del espacio. En Horizontes culturales: Las fronteras de la Ciencia. Madrid: Espasa-Calpe, pp. 207-220.

Millionschikov, M. (1974). El examen decisivo en la historia de la humanidad. En Academia de Ciencias de la Unión Soviética. La Revolución tecnocientífica: Aspectos y perspectivas sociales. Moscú: Progreso, pp. 11-27.

Mora, V. (1985). La ciencia soviética hoy. Conversaciones en la URSS. Moscú: Progreso.

Paluzíe, A., dir. (1984). Astronomía. Explorando el universo. Barcelona: Ramón Sopena.

Parra, J. (2013). Principales problemas de la economía soviética y su incidencia en el final de la URSS. Recuperado de: http://www.rebelion.org/docs/169450.pdf.

Pashuto, V.; Itenberg, B.; Tarnovski, K.; Poletayev, V.; Kumanev, G. y Sharikov, P. (1985). Historia ilustrada de la URSS. Moscú: Editorial de la Agencia de Prensa Nóvosti.

Pedroso, E. (2004). Breve historia del desarrollo de la Ciencia de la Información. En ACIMED 12 (2), pp. 1-10.

Ponomariov, B.; Gromyko, A. y Jvostov, V. (1974). Historia de la política exterior de la URSS. 1945-1970. Moscú: Progreso.

Popov, G. (1988). Desde el ángulo del economista (Acerca de la novela "Nuevo nombramiento" de Alexandr Bek). En El fenómeno Stalin. Moscú: Editorial de la Agencia de Prensa Nóvosti, pp. 12-25.

\section{(c) (i) (2)}

La Revista Estudios es editada por la Universidad de Costa Rica y se distribuye bajo una Licencia Creative Commons Atribución-NoComercial-CompartirIgual 3.0 Costa Rica. Para más información envíe un mensaje a revistaestudios.eeg@ucr.ac.cr. 
R. de E. (1959). La enseñanza de Ciencias e Ingeniería en la Unión Soviética. En Revista de Educación XXXII (92), pp. 40-42. Recuperado de https://www.mecd.gob.es/dctm/revista-de-educacion/1959/1959-

091/1959re91 informacionextranjera.pdf?documentld=0901e72b8189a081 .

Relación de ganadores de los Premios Nobel (1901-1998) (1999). En Cuadernos de Historia de la Salud Pública 86, pp. 51-82. Recuperado de http://bvs.sld.cu/revistas/his/vol 2 99/his11299.pdf.

Reuters (2017, junio 4). El Mar Aral no tiene la batalla perdida. En La Vanguardia (España). Recuperado de http://www.lavanguardia.com/natural/tuhuella/20170603/423158289720/mararal.html.

Rothbard, M. N. (2015). Ciencia, tecnología y gobierno. Auburn (Alabama): Ludwig Von Mises Institute. Recuperado de https://mises.org/files/ciencia-tecnología-ygobiernopdf/download?token=a7UhBL-z.

Saldaña, J. J., comp. (1989). Introducción a la teoría de la historia de las ciencias. México: UNAM.

Saldaña, J. J. (1996). Teatro científico americano. Geografía y cultura en la historiografía latinoamericana de la Ciencia. En Saldaña, J. J. (comp.). La historia social de las ciencias en América Latina. México: Porrúa, pp. 7-41.

Service, R. (2010). Historia de Rusia en el siglo XX. Barcelona: Crítica.

Seton-Watson, H. (1955). La decadencia de la Rusia imperial. 1855-1914. México: Editorial Guarania.

\section{(c) (i) (2) (-)}

La Revista Estudios es editada por la Universidad de Costa Rica y se distribuye bajo una Licencia Creative Commons Atribución-NoComercial-CompartirIgual 3.0 Costa Rica. Para más información envíe un mensaje a revistaestudios.eeg@ucr.ac.cr. 
Stoletov, V (1970). La educación clave de la transformación de la sociedad. En El Correo de la UNESCO XXIII, julio, pp. 12-15.

Stroganov, (2015, 5 febrero). ¿Por qué se fundó la Universidad de la Amistad de los Pueblos? En Russia Beyond. Recuperado de https://es.rbth.com/cultura/2015/02/05/por que se fundo la universidad de la a mistad de los pueblos 47027.

Tapeznikov, S. (1974). El leninismo y la revolución tecnocientífica contemporánea. En Academia de Ciencias de la Unión Soviética. La Revolución tecnocientífica: Aspectos y perspectivas sociales. Moscú: Progreso, pp. 57-80.

Ter-Gazarian, A. (2014, 27 setiembre). La malograda historia de la informática en la URSS. En Russia Beyond. Recuperado de https://es.rbth.com/cultura/technologias/2014/09/27/la_malograda_historia_de_la_i nformatica_en_la_urss_43873.

Timofeichev, A. (2017, 10 noviembre). De Stalin a Putin. ¿cuáles son los premios más destacados de la historia de Rusia? En Russia Beyond. Recuperado de https://es.rbth.com/historia/79552-premios-destacados-historia-rusia.

Valdés, C. y Valdés, J. E. (2014). Boris Gnedenko y las aplicaciones de la teoría de probabilidades. En Estadística española 56 (184), pp. 259-274.

Viales, R. (2012). De la economía mundial a la economía global. En Díaz, D.; Viales, R. y Marín, J. J. Historia de la Cultura. San José: EUNED, pp. 151-314.

\section{(C) $(00)$}

La Revista Estudios es editada por la Universidad de Costa Rica y se distribuye bajo una Licencia Creative Commons Atribución-NoComercial-CompartirIgual 3.0 Costa Rica. Para más información envíe un mensaje a 
Revista Estudios, (36), 2018.

Junio 2018-Noviembre 2018

ISSN 1659-3316

Díaz Bolaños Ronald Eduardo

Wetter, G. A. (1968). Filosofía y ciencia en la Unión Soviética. Madrid: Guadarrama.

Wozniak, J. (2013). La hambruna ucraniana de 1932-1933 como caso de genocidio. Una introducción al debate. En Revista Genocidio 5, pp. 49-64. Recuperado de http://revistagenocidio.com.ar/wp-content/uploads/2013/05/049-a064.pdf.

Zugaza, S. (2012, 5 mayo). La librería rusa de Lima. En Russia Beyond. Recuperado de https://es.rbth.com/articles/2012/05/05/la_libreria_rusa_de_lima_17052 